\title{
Teenagers' use of sexual health services: perceived need, knowledge and ability to access
}

\author{
Alison Parkes, MA, PhD, Researcher; Daniel Wight, BA, PhD, Senior Researcher; Marion Henderson, BA, Senior Researcher, \\ Medical Research Council Social and Public Health Sciences Unit, University of Glasgow, Glasgow, UK
}

Correspondence: Alison Parkes, MRC Social and Public Health Sciences Unit, University of Glasgow, 4 Lilybank Gardens, Glasgow G12 8RZ,UK.E-mail:alison-p@msoc.mrc.gla.ac.uk

(Accepted 4 June 2004)

Journal of Family Planning and Reproductive Health Care 2004; 30(4): 217-224

\begin{abstract}
Introduction An individual teenager's use of services may depend on perceived need, on knowledge of sexual health and local services, and on ability to access. This paper presents the first UK large-scale quantitative analysis of these factors, comparing those who use services with those who do not.

Methods 15/16-year-olds $(n=5747)$ were questioned about their use of sexual health services in the SHARE trial of a school sex education programme in 25 schools in Lothian and Tayside, Scotland, UK. Multilevel statistical models examined the role of different factors on service use.

Results One-third of teenagers had used a service, and use was strongly related to sexual experience. In addition, some family influences and being a school leaver were associated with service use, although we found no evidence for class, ethnic or religious barriers to use. Proximity to specialist clinics was linked with greater use, while low spending money and high parental monitoring were associated with less use. Teenagers with better knowledge, who rated their school sex education as effective, who were comfortable talking about sex and who had discussed contraception with peers were more likely to have used services. Differences in use relating to sexual experience, knowledge, feeling comfortable talking about sex and talking with peers helped to explain gender differences in service uptake.

Conclusion There is potential to influence service use through better knowledge and confidence imparted through school sex education, and by improving the links between services and schools.
\end{abstract}

\footnotetext{
Key message points

- Sexual experience, proximity to clinics, parental influences, knowledge and confidence were all associated with service use/non-use.

- Effective school sex education was associated with increased uptake of services.

- Boys' uptake of services may be improved through better knowledge, greater confidence and peer group discussion.

\section{Introduction}

Current UK policies aimed at increasing uptake and improving delivery of sexual health services among teenagers view availability, confidentiality and nonjudgemental staff as key features of these services. ${ }^{1-3}$ These supply-side attributes may encourage teenagers embarking on sexual relationships to use services, but individual uptake will also depend on perceived need for help, knowledge of local services and ability to access them. Teenagers in short-term relationships may feel less need to use services. ${ }^{4,5}$ Girls need to use services more than boys, perhaps because of greater concerns over pregnancy, but also because the pill is obtainable only on prescription.
}

Boys prefer to buy condoms $\mathrm{s}^{7,8}$ and to obtain advice from helplines or websites. ${ }^{9}$ There is scope to influence perceived need through better sexual health knowledge ${ }^{10}$ and information about local services. Many teenagers appear to hear about services from their friends rather than via publicity material. ${ }^{11-13}$

Teenagers' ability to access services is likely to depend on a number of interrelated factors. The first group is linked to where they live or go to school in relation to different services. Proximity may be important for rural teenagers 14 who also have fewer service options available to them; 15 although both rural and urban teenagers may prefer to travel to more distant services that provide greater anonymity. ${ }^{16,17}$ There is debate over whether all types of existing sexual health provision are equally suitable for young people. With teenagers' concerns over general practitioners' (GPs') respect for confidentiality, ${ }^{18,19}$ family planning clinics (FPCs) may be preferred because they appear to offer a more anonymous, confidential, nonjudgemental specialist service. ${ }^{20,21}$ A specialist youth service may be teenagers' ideal model of service provision, ${ }^{11,22-24}$ although increasing FPC hours coupled with targeted outreach to schools dramatically increased young people's use of a mainstream service. ${ }^{13}$ Some young peoples' services are more attractive to boys than mainstream services ${ }^{25-27}$ but not all. 11,28

A second group of factors affecting ability to access services includes cultural and language barriers, ${ }^{29,30}$ family influences - for example, fear of parental disapproval ${ }^{14}$ - and individual confidence in discussing sensitive issues. 31

Studies of teenagers' use of sexual health services have typically been small-scale surveys and qualitative research. Profiles of those using particular types of service have not compared them with non-users or those visiting other services; and research on teenagers' views of an ideal service has not related these to actual patterns of use. We know relatively little in the UK about what, in practice, stimulates or discourages teenagers to use sexual health services of all types. The aim of this paper is to explore the hypothesis that use of sexual health services will depend on a range of factors related to perceived need, knowledge and ability to access. This study is the largest UK quantitative analysis to compare teenage users and non-users of services, examining the role of sociodemographic background, sexual experience, access, knowledge and school sex education.

\section{Methods}

Sample

Following approval by Glasgow University's Ethical Committee for Non-Clinical Research Involving Human Subjects, all 47 non-denominational state secondary schools within $24 \mathrm{~km}$ of the main cities in Lothian and Tayside, Scotland, UK were invited to participate in the 
Table 1 Service use by teenagers aged 15/16 years in the SHARE dataset ${ }^{a}$

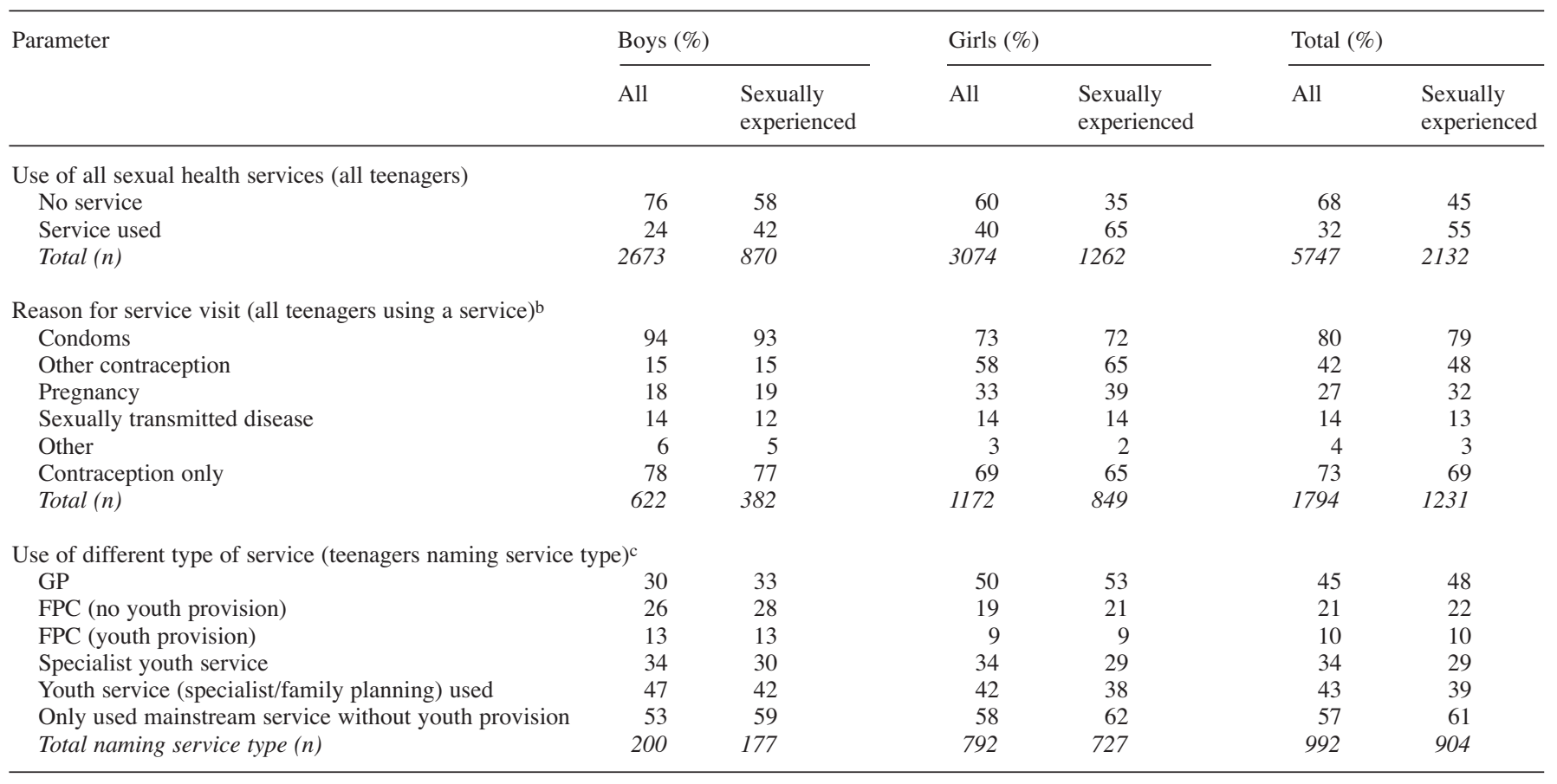

aPercentages used data weighted for attrition at follow-up, frequencies used unweighted data.

bServices could be used for more than one reason, so percentages sum to more than 100 .

${ }^{\mathrm{c}}$ More than one type of service may be used. Service types used by more than 5\% of teenagers shown in first four rows.

FPC, family planning clinic; GP, general practitioner.

SHARE (Sexual Health and Relationships: Safe, Happy and Responsible) controlled trial of school sex education, 32,33 with the exception of five pilot schools. Schools in the SHARE trial were allocated either the SHARE programme of sex education or continuing with their existing sex education. The SHARE programme consisted of a 5-day teacher training programme and a 20 session pack for third- and fourth-year students. Parents were given the opportunity to withdraw their children, and pupils were also given the option to withdraw or omit questions at any stage. At baseline (age 13/14 years), three pupils from the 24 schools who elected to join the study at baseline were excluded by teachers because of learning difficulties, seven pupils were withdrawn by their parents, and 32 pupils chose not to participate. Taking into account these opt-outs and absences from school, the participation rate at baseline was $94 \%$.

This paper is based on data from a follow-up selfcompleted questionnaire administered by researchers under examination conditions at age 15 or 16 years (average age, 16 years 1 month), by which stage another school had joined the study $(n=5747)$. The response rate was $69 \%$ of the total eligible sample, the shortfall being mainly attributable to lower participation among school leavers completing postal questionnaires. Only $2 \%$ of those still at school chose to opt out. The analysis used weighted data to compensate for the greater attrition for boys, lower social class and those reporting sex at baseline, maintaining the representativeness of the sample compared to the 1991 census that was established at baseline. ${ }^{2,34}$

Respondents were asked whether they had used a sexual health service of any kind (including GP services) in the previous 2 years, for each of five different purposes (for condoms, other contraceptives, advice about pregnancy, advice about sexually transmitted diseases or 'other' reasons), and for the name of the service used. From previous research ${ }^{35}$ and teenagers' replies to the questionnaire, 39 sexual health clinics were identified in the study area in addition to GP services at the time of data collection (1996-1999). Ten of these were youth services: most were designated times for young people within FPCs, and four were exclusively for young people. The two most popular were the Brook Advisory Centre in Edinburgh (now Caledonia Youth) and The Corner, a multipurpose, drop-in centre in Dundee.

Statistical modelling of service use

Exploratory bivariate analysis of the data was performed using SPSS version 11.5 (SPSS Inc., Chicago, IL, USA). At this stage, existing research described in the Introduction and factors related to experience of sexual intercourse in the SHARE dataset ${ }^{36,37}$ were used to inform the choice of variables that might be associated with service use. In the next stage, multilevel logistic regression models of service use were fitted using iterative generalised least squares with a second-order Taylor series expansion and penalised quasi-likelihood in MLWiN. ${ }^{38}$ There were two levels in the models: individuals and schools. Dummies were included for missing values of the variables, so the same individuals were included in all models. Each model routinely controlled for cohort and age at questionnaire.

The first three stages of the analysis focused on individual-level predictors of service use. The final stage

Table 2 Service use by teenagers aged 15/16 years in the SHARE dataset: use of youth (specialist/family planning) services by purpose of visit (all teenagers naming service type)

\begin{tabular}{lcccccc}
\hline & Condoms & Other contraception & Pregnancy & Sexually transmitted disease & Other & All reasons for visit \\
\hline Youth service ever used (\%) & 56 & 29 & 46 & 47 & 38 & 43 \\
Mainstream service only (\%) & 44 & 71 & 54 & 53 & 62 & 57 \\
Total naming service type $(n)$ & 617 & 551 & 298 & 106 & 72 & 992 \\
\hline
\end{tabular}


Table 3 Individual effects on sexual health service use at age 15/16 years: sociodemographic variables and sexual relationships $(n=5747)$

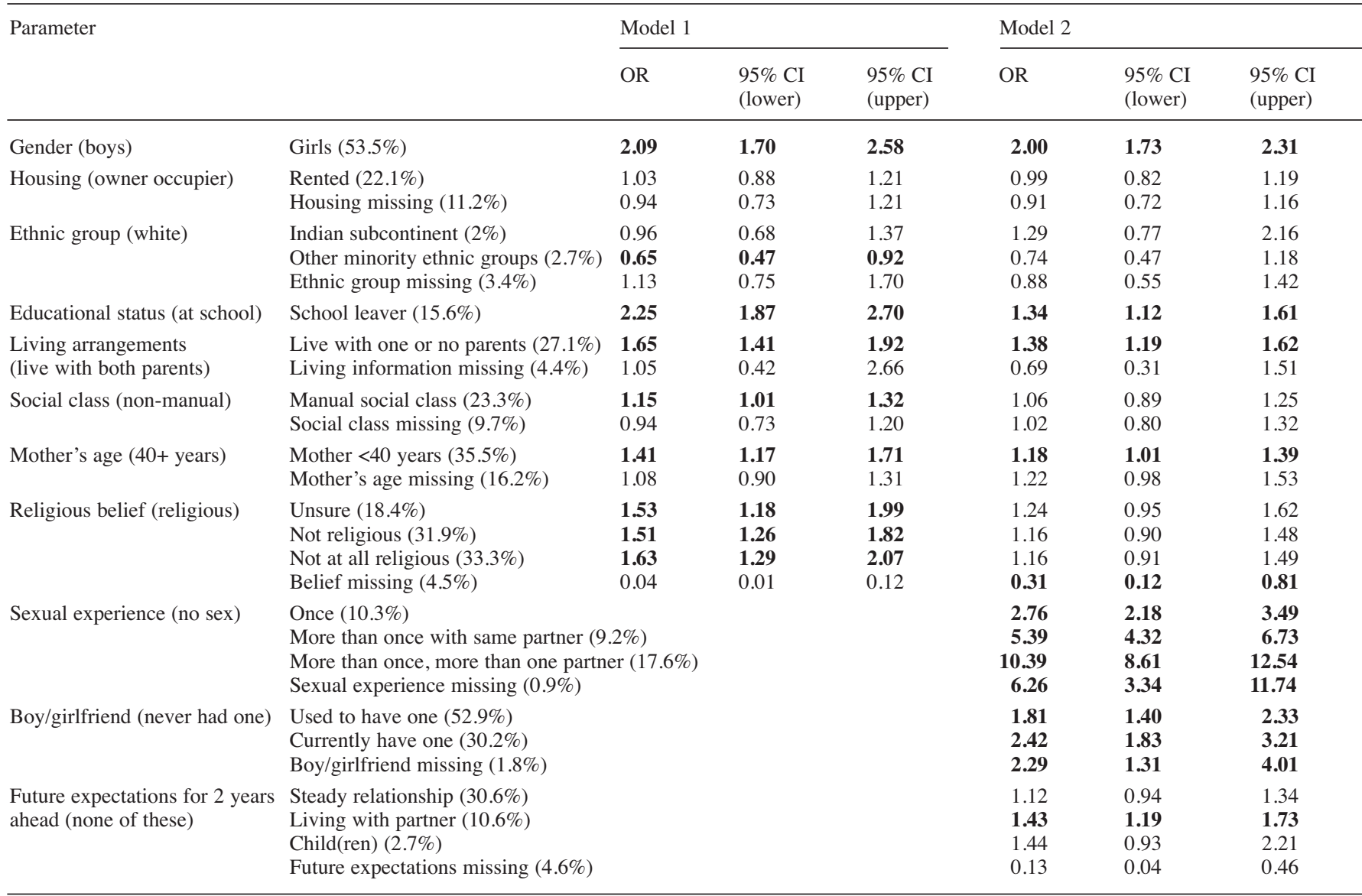

Models control for age and cohort. Column 1 shows reference category in parentheses. Percentages indicate share of contrast category in sample.

Bold type denotes OR significant at $\mathrm{p}<0.05$ or less. CI, confidence interval, OR odds ratio.

focused on trying to explain the remaining between-school variation in service use, with a combination of individuallevel and school-level variables.

Model 1 incorporated sociodemographic predictors of service use, identified through bivariate analysis: gender, social class, housing tenure, family structure, mother's age, ethnic group and religiosity.

Model 2 incorporated sociodemographic predictors as for Model 1, plus experience of sexual intercourse, boy/girlfriend and future expectations concerning relationships. At this stage, some sociodemographic predictors of service use in Model 1 became insignificant, because they helped predict sexual experience. ${ }^{36,37}$ These were dropped before progressing to Model 3.

In Model 3, variables related to teenagers' ability to access clinics, confidence, knowledge and attitudes were added.

Postcodes were used to calculate straight-line distances between pupils' home address and their nearest undifferentiated and youth-specific sexual health clinics. Proximity to GPs was not used, but this is more uniform. The mean distance from home to the nearest clinic was $2.9 \mathrm{~km}$, and to the nearest youth service was $7.2 \mathrm{~km}$. The majority of pupils $(60 \%)$ were within $3 \mathrm{~km}$ of a clinic of any type and $27 \%$ were within $3 \mathrm{~km}$ of a youth clinic. While fewer than one in ten pupils lived more than $10 \mathrm{~km}$ from a clinic of any type, around four in ten pupils lived more than $10 \mathrm{~km}$ from a youth clinic. Straight-line distances were transformed to standardised z-scores based on normal distributions, which were used as indexes of relative clinic proximity in the models.

Other variables that might have related to teenagers' ability to access clinics were levels of parental monitoring (mean scores for four questions concerning rules about going out in the evening ${ }^{39}$ ) and spending money. Confidence was measured through self-esteem (mean scores for four questions) and how comfortable teenagers felt when talking about sex with others (mean scores for seven questions). There were two variables for knowledge, namely knowledge of sexual health (mean scores for eight questions) and knowledge of different places where contraception may be obtained on prescription. Other variables included whether teenagers had talked to friends about using contraception in the past year (from two questions about condoms and contraception in general) and whether they considered it important to plan protection against pregnancy/sexually transmitted diseases (two questions).

Significant school-level variance in Model 3 suggested that a school-level effect on service use remained. Variance significance is given by reference to its standard error in a one-sided test, a value greater than $1.64 \times$ the standard error (SE) is significant at $\mathrm{p}<0.05 .40$

In Model 4, four variables were added to see whether there was any reduction in school-level variance: school-level deprivation, school proximity to clinics, and individual opinions of both the coverage and effectiveness of school sex education. School-level deprivation was added because of its association with between-school variation in sexual experience. ${ }^{34}$ This measure incorporates local unemployment rates, area deprivation score, pupils' post-school destination, paid school meals, staying on rates and attendance rates. 35 School straight-line distances to nearest clinic/youth clinic were used in a similar way to distances from home to clinic. The mean minimum distance from school to all types of clinic was $2.1 \mathrm{~km}$, and to a youth clinic was 7.0 $\mathrm{km}$, similar to the mean distances from home. 


\section{Making Sure Contraception Comes First}

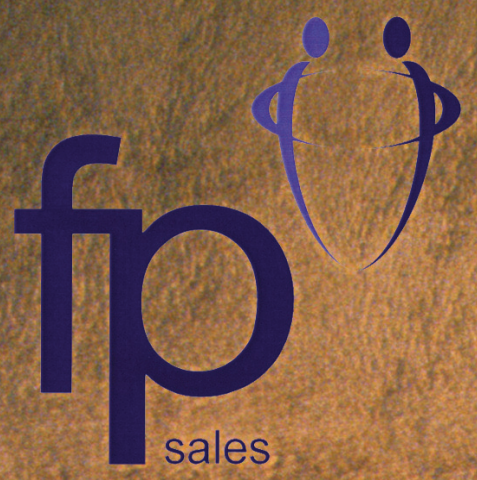

Special offers:

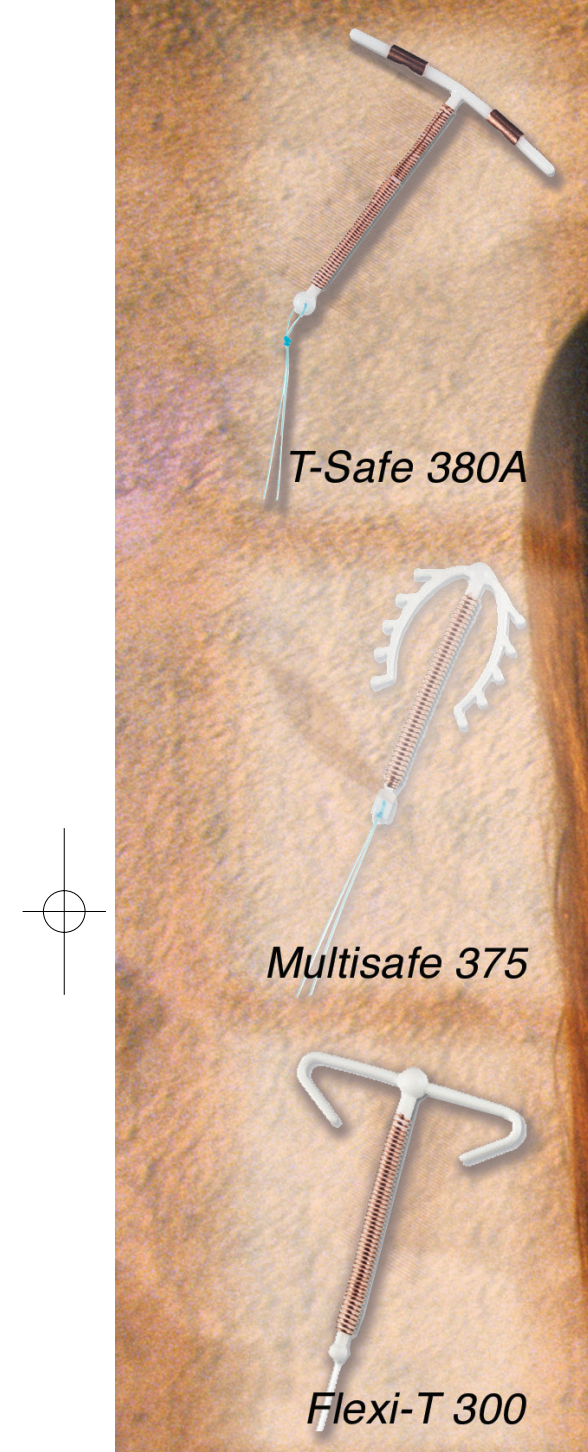

Flexi-T 380 Now Avalle:

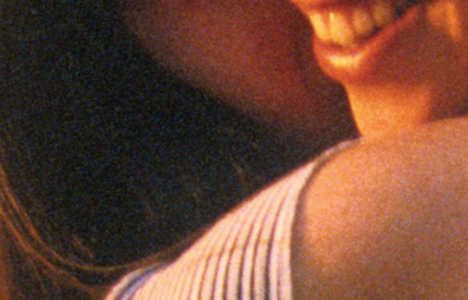

NOVEMBER: Buy a pack of

OCTOBER: Buy pack of T-Safe (A19) for $£ 94.00$ and get a pack of thread retrievers (D520) FREE

worth $£ 10.80$
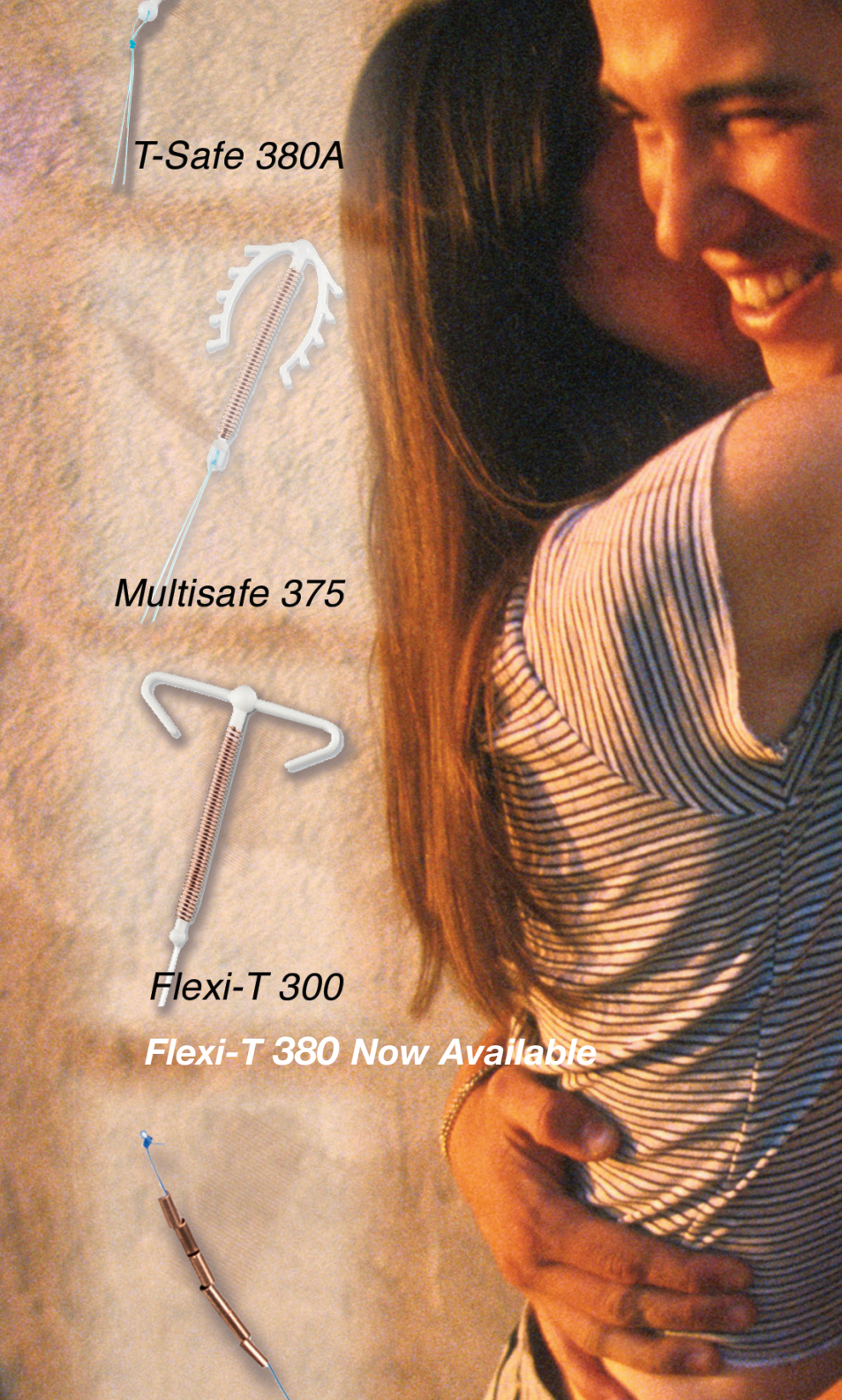

Gyneiix

08704424061 www.fpsales.co.uk 10 Neo-Safe (A2510) for $£ 82.71$ and get 200 gloves (A2510) FREE worth $£ 8.60$

DECEMBER: Buy 4 Gynefix (A200) for $£ 99.00$ and get a box of 25 disposable Speculum (D5259) $1 / 2$ price saving $£ 17.92$

Please Quote JFP1004 To qualify for special offers.

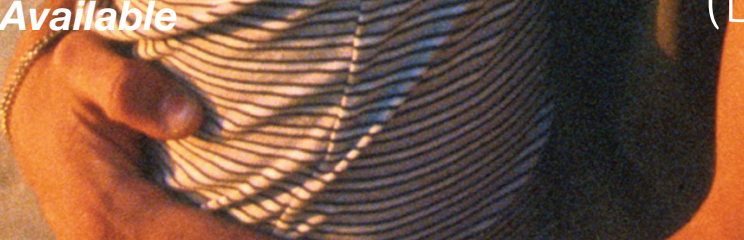


Table 4 Individual effects on sexual health service use at age 15/16 years: sociodemographic variables, sexual relationships, access, knowledge, confidence and attitudes $(n=5747)$

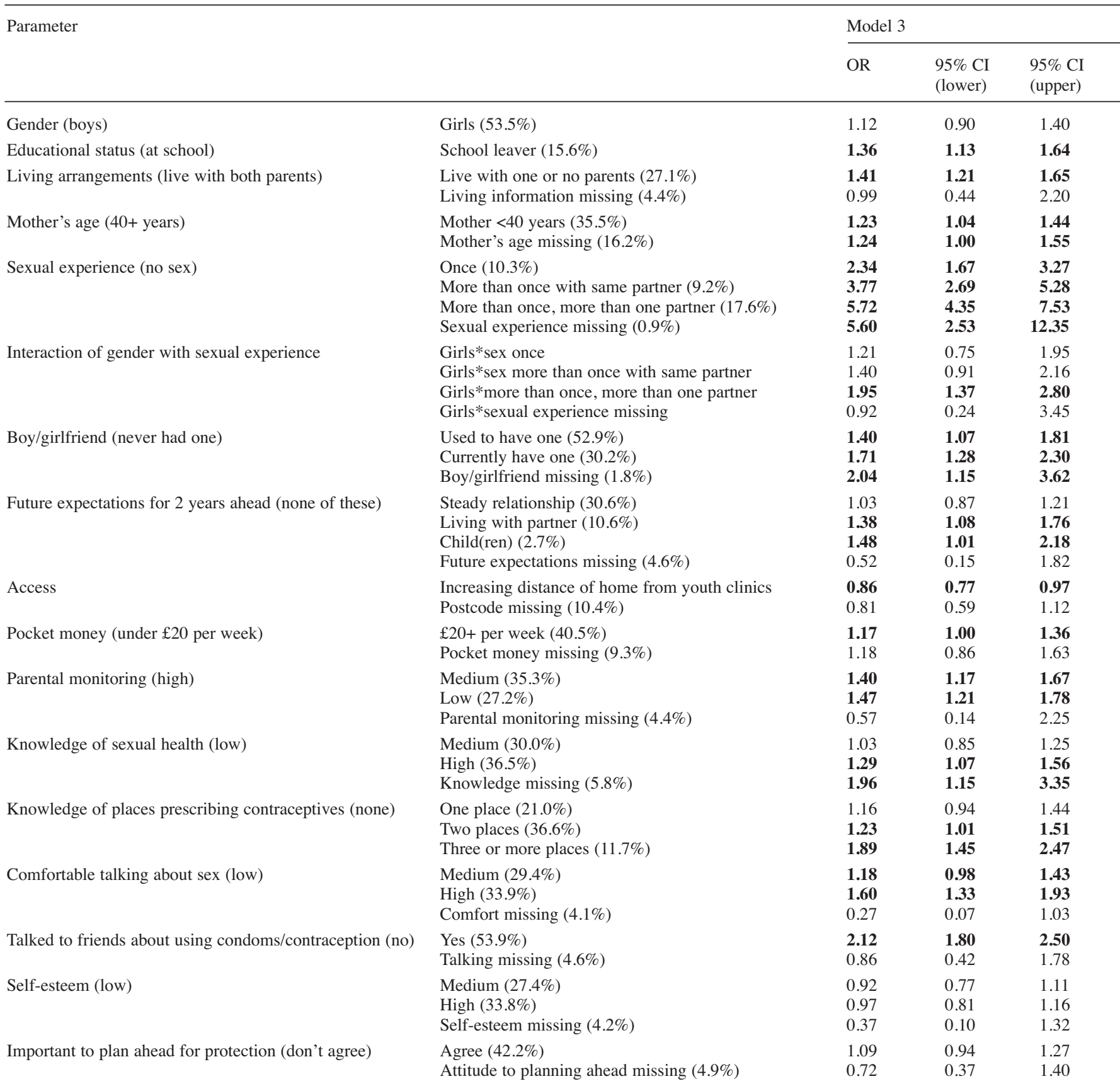

Models control for age and cohort. Column 1 shows reference category in parentheses. Percentages indicate share of contrast category in sample. Asterisk denotes interaction. Bold type denotes OR significant at $\mathrm{p}<0.05$ or less. CI, confidence interval, OR odds ratio.

Teenagers' opinions of effectiveness and coverage of school sex education were factor scores from 11 questions.

The analysis thus examined separate blocks of variables in an attempt to identify important independent predictors of service use from four main areas identified in the literature on sexual health services for young people: sociodemographic; relationships; access, knowledge and confidence; and school.

\section{Results}

Table 1 shows that $32 \%$ of all teenagers had used a service, although use was higher for girls $(40 \%)$ than boys $(24 \%)(\mathrm{p}<0.001)$. Among teenagers who reported sexual intercourse by age $15 / 16$ years, $55 \%$ had used a service, again more girls (65\%) than boys $(42 \%)$ $(\mathrm{p}<0.001)$. A bigger majority of boys $(78 \%)$ than of girls $(69 \%) \quad(\mathrm{p}<0.001)$ used services to obtain contraception only, most boys going exclusively for condoms. Advice about pregnancy and sexually transmitted diseases was sought by $27 \%$ and $14 \%$ of teenagers, respectively.

Girls were more likely to provide detailed information (68\%) on service type than boys (32\%), preventing us from establishing whether boys have a differential preference for youth services. Of those who provided the information, $43 \%$ reported using youth services, with no significant variation by gender. Among those using mainstream services with no youth provision, more girls $(50 \%)$ than boys $(30 \%)(\mathrm{p}<0.001)$ used a GP, while more boys (26\%) than girls (19\%) $(\mathrm{p}<0.05)$ used family planning services. Teenagers who wanted contraceptives other than condoms (mainly the pill) were more likely to have used mainstream services, 
Original Article

Table 5 Gender differences in parental monitoring, knowledge and talking about sex

\begin{tabular}{|c|c|c|c|c|c|c|}
\hline Parameter & & \multicolumn{2}{|l|}{ Boys } & \multicolumn{2}{|l|}{ Girls } & $\mathrm{p}$ \\
\hline Parental monitoring & $\begin{array}{l}\text { Low/medium } \\
\text { High }\end{array}$ & $\begin{array}{r}1885 \\
662\end{array}$ & $\begin{array}{l}74.0 \\
26.0\end{array}$ & $\begin{array}{l}1704 \\
1242\end{array}$ & $\begin{array}{l}57.8 \\
42.2\end{array}$ & $<0.001$ \\
\hline Knowledge of sexual health & $\begin{array}{l}\text { Low/medium } \\
\text { High }\end{array}$ & $\begin{array}{r}1757 \\
736\end{array}$ & $\begin{array}{l}70.5 \\
29.5\end{array}$ & $\begin{array}{l}1557 \\
1364\end{array}$ & $\begin{array}{l}53.3 \\
46.7\end{array}$ & $<0.001$ \\
\hline Knowledge of places prescribing contraceptives & $\begin{array}{l}0-2 \text { places } \\
3+\text { places }\end{array}$ & $\begin{array}{r}2498 \\
175\end{array}$ & $\begin{array}{r}93.5 \\
6.5\end{array}$ & $\begin{array}{r}2574 \\
500\end{array}$ & $\begin{array}{l}83.7 \\
16.3\end{array}$ & $<0.001$ \\
\hline Comfortable talking about sex & $\begin{array}{l}\text { High/medium } \\
\text { Low }\end{array}$ & $\begin{array}{l}1482 \\
1072\end{array}$ & $\begin{array}{l}58.0 \\
42.0\end{array}$ & $\begin{array}{r}2156 \\
800\end{array}$ & $\begin{array}{l}72.9 \\
27.1\end{array}$ & $<0.001$ \\
\hline Talked with friends about using condoms/contraceptives & $\begin{array}{l}\text { No } \\
\text { Yes }\end{array}$ & $\begin{array}{l}1525 \\
1015\end{array}$ & $\begin{array}{l}60.0 \\
40.0\end{array}$ & $\begin{array}{r}860 \\
2085\end{array}$ & $\begin{array}{l}29.2 \\
70.8\end{array}$ & $<0.001$ \\
\hline
\end{tabular}

while teenagers who wanted condoms were more likely to use youth services, suggesting that youth services may have catered more for boys than mainstream services (Table 2).

The overall rate for service use for the modelling sample, after controlling for age at questionnaire and cohort, was found to be $33 \%$ with $95 \%$ confidence interval (CI) $22 \%, 46 \%$ (Table 1 not adjusted for age and cohort). The between-schools variance in service use accounted for $4 \%$ of the total variance $(\mathrm{p}<0.001)$

The bivariate exploratory analysis found that all variables considered were associated with service use $(p<0.05$ or lower), with the exception of the six-fold urban-rural Scottish Household Survey classification for home postcode 41 and the SHARE sexual health education programme intervention. These two variables were not included in the multivariate analysis. In the following description of the results of multivariate Models 1 through 4 , the level of significance used is $\mathrm{p}<0.05$ or lower.

Table 3 shows sociodemographic variables associated with increased use of services (Model 1, first three columns): being a school leaver, female, not living with both parents, low religiosity, having a young mother and with parents in manual social classes. Those in minority ethnic groups (other than from the Indian subcontinent) were less likely to use services.

Controlling for sexual experience in Model 2 (Table 3, last three columns) resulted in some of these variables losing significance. School leavers, those not living with both parents and having a young mother remained significant at the $p<0.05$ level.

The amount of sexual experience was a strong predictor

Table 6 Comparison of school-level variance in different models

\begin{tabular}{|c|c|c|c|c|c|}
\hline & Null model & Model 1 & Model 2 & Model 3 & Model 4 \\
\hline \multirow{2}{*}{$\begin{array}{l}\text { School-level variance } \\
\text { (SE) }\end{array}$} & 0.154 & 0.133 & 0.163 & 0.183 & 0.111 \\
\hline & 0.050 & 0.045 & 0.056 & 0.062 & 0.041 \\
\hline \multirow[t]{12}{*}{ Variables included } & Age and cohort & Age and cohort & Age and cohort & Age and cohort & Age and cohort \\
\hline & & Sociodemographics & Sociodemographics & $\begin{array}{l}\text { Reduced set of } \\
\text { sociodemographics }\end{array}$ & $\begin{array}{l}\text { Reduced set of } \\
\text { sociodemographics }\end{array}$ \\
\hline & & & Sexual experience & Sexual experience & Sexual experience \\
\hline & & & $\begin{array}{l}\text { Anticipated sexual } \\
\text { relationship }\end{array}$ & $\begin{array}{l}\text { Anticipated sexual } \\
\text { relationship }\end{array}$ & $\begin{array}{l}\text { Anticipated sexual } \\
\text { relationship }\end{array}$ \\
\hline & & & & Access & Access \\
\hline & & & & Confidence & Confidence \\
\hline & & & & Knowledge & Knowledge \\
\hline & & & & Attitudes & Attitudes \\
\hline & & & & & School-level deprivation \\
\hline & & & & & School-level proximity \\
\hline & & & & & to clinics \\
\hline & & & & & $\begin{array}{l}\text { Individual opinions of } \\
\text { school sex education }\end{array}$ \\
\hline
\end{tabular}

SE, standard error. teenagers (data not shown) found that those who reported only having had sexual intercourse once were significantly less likely to have used services than those with more than one experience, suggesting that teenagers delayed using services until their sexual behaviour was more established.

Controlling for sexual experience, it appeared that those who anticipated an enduring sexual relationship were also more likely to have used a service: those with a current or previous boy/girlfriend, and those that expected to be living with a partner in the future, were both more likely to have used a service.

Some of the dummies for missing values in Model 2 (religious belief, sexual experience and boy/girlfriend) showed significant positive associations with service use. This may be because teenagers in these groups were more sexually involved than their family background would permit, and were reluctant to divulge sensitive information.

The interactions between gender and sexual experience were explored for Model 2. It was found that girls with more than one sexual partner were almost twice as likely to have used a service than boys with more than one partner [odds ratio (OR) $1.94,95 \%$ CI 1.37-2.75]. This may reflect girls' greater concern with the risk of pregnancy from increased sexual activity, although even with the interactions, girls were still more likely to use services (OR reduced to $1.64,95 \%$ CI 1.31-1.95). Boys may have less need to use services, since they can buy condoms whereas the pill is only available on prescription. Incorporating a variable indicating whether a teenager had bought condoms in the previous year (data not shown) did not, however, predict lower service use, even when an interaction effect 
Table 7 School factors and use of sexual health services by teenagers at age 15/16 years

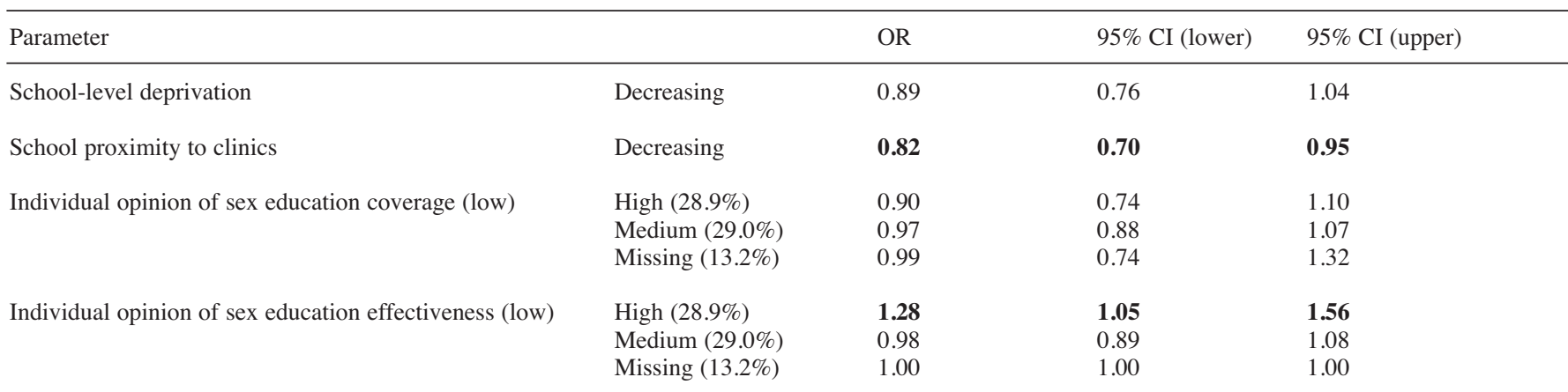

Models control for variables in Model 3. Column 1 shows reference category in parentheses. Percentages indicate share of contrast category in sample. Bold type denotes OR significant at $\mathrm{p}<0.05$ or less. CI, confidence interval, OR odds ratio.

with gender was included. Thus gender differences in the propensity to contact services with increased sexual experience and in sources for contraceptives did not appear to account for all of the gender difference in service use.

Model 3 (Table 4) suggested that variables related to better information and ease of access were associated with greater service use. Better knowledge of sexual health and places where contraception is prescribed, talking with friends about contraception, and medium/high levels of comfort in talking about sex were all linked with service use, as was proximity of home to youth services rather than to clinics in general (data not shown, OR $0.93,95 \%$ CI $0.85-1.02$ ), high spending money and medium/low levels of parental monitoring. However self-esteem and attitudes towards planning protection were not significant. As for Model 2, some of the dummies for missing values showed a significant positive association with service use (in particular, missing sexual experience).

The OR for girls using a service was reduced and no longer significant in Model 3, suggesting that some of the variables added in Model 3 helped to explain gender differences in service use. Despite the fact that boys were less likely to experience high levels of parental monitoring than girls, which would predict higher levels of service use, boys were less knowledgeable than girls, more likely to feel uncomfortable talking about sex than girls and were less likely to have talked about contraceptives with their friends than girls (Table 5).

In Model 4, with the addition of school characteristics there was a marked reduction in the school-level variance to below the level in the null model (Table 6). In this final model, R-square calculated following Snijders and Bosker ${ }^{40}$ was 0.46 . Increased school-level deprivation was not significantly associated with service use. Reduced school proximity to clinics was associated with a significant reduction in service use, with an OR similar to that in Model 3 for home-youth clinic distance. Because of its covariance with the home-youth clinic distance, the latter was omitted from Model 4. School-youth clinic distance did not help to predict service use (data not shown, OR 0.95, 95\% CI 0.81-1.12). Teenagers with high opinions of the coverage of school sex education were no more likely to visit services, although those with high opinions of their sex education's effectiveness were more likely to have used a service (Table 7).

\section{Discussion}

Our study confirmed the hypothesis that perceived need, knowledge and ability to access are all important factors in shaping patterns of service use. Although we lack information on the timing of service visits in relation to sexual intercourse, it was apparent that many teenagers delayed using services until they had intercourse more than once, or with more than one partner. Encouraging teenagers to access services earlier may be achieved through improving knowledge about sexual health and service provision, since these were associated with service use. Information may come through a number of different channels: teenagers who had talked about contraception with their friends, who were comfortable talking about sex and those who rated their school sex education as highly effective were all more likely to use services. However, these variables may also reflect the positive effects of education and discussion on shaping norms and confidence to use services. Our analysis suggests that more effort needs to be devoted to enhancing boys' knowledge, confidence and talking with peers, as these factors helped to explain gender differences in service use in our models. Questions as to whether boys are as receptive to sex education as girls need to be set against our finding that boys gave higher ratings for school sex education coverage and effectiveness than girls. Although in a cross-sectional study causation is not clear, additional analysis of our data (not presented here) suggested that increased knowledge, talking with friends about contraception, feeling comfortable talking about sex and perceived effective school sex education among nonservice users at age 15/16 years was associated with greater anticipated ease of future contact with sexual health services. This study found no evidence for a direct effect of the SHARE sex education programme on service use in bivariate exploration, but the programme may have indirectly promoted service uptake by increasing sexual health knowledge and raising the rating of school sex education. ${ }^{32}$

Physical proximity was one factor that influenced teenagers' ability to access clinics. Distance from home to youth clinics may be more critical than home distance to all clinics, if youth clinics are accessed mainly at weekends or evenings, and if teenagers prefer to use this type of clinic if it is within their home territory, ${ }^{42}$ perhaps going with local friends. Conversely, the effect of school proximity to all specialist sexual health clinics on overall service use may reflect teenagers travelling directly there from school. Although our information on use of different service types was limited by low reporting among boys, exploratory modelling found that talking with friends about contraception and increased school proximity to youth clinics were significant factors predicting a preference for youth over mainstream services.

We found no evidence for ethnic, religious or class barriers to service use after controlling for sexual activity. School leavers, those in households with only one/no parent present, those with younger mothers and those with lower parental monitoring were more likely to use services, which may relate to lower parental surveillance. 
School-based clinics might help teenagers to use services more independently of family influences, and better links between school and medical staff may also increase service uptake. These may take the form of better information about local services at school; involving health service staff in sex education programmes; using school nurses or teachers to book appointments at services on behalf of teenagers; arranging school visits to clinics and trying out mock classroom clinics. ${ }^{2,43-47}$ However, our research also suggests that talking with friends may be important, lending support to peer delivery of sex education to complement more traditional teacher-based methods (see, for example, the studies by Mellanby et al. ${ }^{48}$ and Forrest et al. ${ }^{49}$ ).

\section{Acknowledgements}

The authors wish to thank Izzy Butcher, Geoff Der, Philip McLoone and Gillian Raab for help with suggestions for the clinic proximity analysis, and Graham Hart for comments on drafts.

\section{Statements on funding and competing interests}

Funding. None identified.

Competing interests. None identified.

References

1 Department of Health. The National Strategy for Sexual Health and HIV. London, UK: Department of Health, 2001.

2 Health Development Agency. Teenage Pregnancy: An Update on Key Characteristics of Effective Interventions. London, UK: Health Development Agency, 2001.

3 Teenage Pregnancy Unit. Best Practice Guidance on the Provision of Effective Contraception and Advice Services for Young People. Effective Contraception and Advice Services for Young People.

4 Jaccard J. Adolescent contraceptive behavior: the impact of the provider and the structure of clinic-based programs. Obstet Gynecol 1996; 88(3 Suppl.): 57S-64S.

5 Stone N, Ingham R. When and why do young people in the United Kingdom first use sexual health services? Perspect Sex Reprod Health 2003; 35: 114-120.

6 Duncan B. Barriers to service use: an exploration of heterosexua men's attitudes to sexual health and sexual health provision. In: Improving the Sexual Health of Men in Scotland. Edinburgh, UK: Health Education Board for Scotland, 2002.

7 Wardle SA, Wright PJ. Family planning services -- the needs of young people: a report from Mid Staffordshire. Br J Fam Plann 1993; 19: $158-160$.

8 Harden A, Ogden J. Sixteen to nineteen year olds' use of, and beliefs about, contraceptive services. Br J Fam Plann 1999; 24: 141-144.

9 Hagley M, Pearson H, Carne C. Sexual health advice centre. Int J Adolesc Med Health 2002; 14: 125-130.

10 Aten MJ, Siegel DM, Roghmann KJ. Use of health services by urban youth: a school-based survey to assess differences by grade level, gender, and risk behavior. J Adolesc Health 1996; 19: 258-266.

11 Morrison A, Mackie CM, Elliott L, et al. The Sexual Health Help Centre: a service for young people. J Public Health Med 1997; 19: $457-463$

12 Morrison C, Bayne D. Safe and Sorted? Not in Leith: An Action Research Project Exploring the Barriers for Young People in Accessing Sexual Health Services. Edinburgh, UK: TASC Agency and Edinburgh Youth Social Inclusion Partnership, 2001

13 Baraitser P, Fettiplace R, Dolan F, et al. Quality, mainstream services with proactive and targeted outreach: a model of contraceptive service provision for young people. J Fam Plann Reprod Health Care 2002; 28: 90-94.

14 Allaby MAK. Reviewing family planning services: a method for population-based outcome-related needs assessment. Br J Fam Plann 1993; 18: 102-105.

15 Butler R, Solomon S. Review of Sexual Health Services for Young People in Scotland. Edinburgh, UK: Health Education Board for Scotland, 2002.

16 Garside R, Ayres R, Owen M, et al. Anonymity and confidentiality: rural teenagers' concerns when accessing sexual health services. J Fam Plann Reprod Health Care 2002; 28: 23-26.

17 McAllister KF, Elliott L, Thomson DAM, et al. Evaluation of a young person's sexual health service in a commercial setting. J Fam Plann Reprod Health Care 2002; 28: 203-206.

18 Lo SV, Kaul S, Kaul R, et al. Teenage pregnancy - contraceptive use and non-use. Br J Fam Plann 1994; 20: 79-83.

19 Burack R. Young teenagers' attitudes towards general practitioners and their provision of sexual health care. Br J Gen Pract 2000; 50(456): $550-554$

20 Allaby MAK. Contraceptive services for teenagers: do we need family planning clinics? BMJ 1995; 310: 1641-1643.
21 Smith M. Why young women in Dublin choose family planning centres for their contraceptive needs. Br J Fam Plann 1996; 22 $145-150$.

22 Smith A. Young people's contraception and sexual health: report of a local needs assessment in Staveley, North Derbyshire. Br J Fam Plann 2001; 27: 29-32.

23 Chambers R, Boath E, Chambers S. Young people's and professionals' views about ways to reduce teenage pregnancy rates: to agree or not agree. J Fam Plann Reprod Health Care 2002; 28 : $85-90$.

24 Beddard D, Chandiok S, James P, et al. A six-month pilot of a collaborative clinic between genitourinary medicine services and a young person's sexual health clinic. J Fam Plann Reprod Health Care 2003; 29(2): 40-42.

25 Bloxham S, Capstick S, Greenwood A. Combining GUM and contraceptive services for young people: profile of an innovative contraceptive services for young peopl
clinic. Br J Fam Plann 1999; 25: 18-21.

26 Edgecombe J, O'Rourke B. Mobile outreach services for young people. Int J Adolesc Med Health 2002; 14: 111-115.

27 Williams EC, Kirkman RJE, Elstein M. Profile of young people's advice clinic in reproductive health, 1988-1993. BMJ 1994; 309: 786-788.

28 Wilson S, Daniel S, Pearson J, et al. An evaluation of a new teenage clinic and its impact on teenage conceptions in Nottingham from 1986 to 1992. Contraception 1994; 50: 77-86.

29 Garg M. Uptake of family planning services among an ethnically mixed population in a general practice setting. Br J Fam Plann 1998 24: 82-83.

30 Patel-Kanwal H. Improving access to sexual health advice for black and minority ethnic young people. Sex Educ Matters 2001; 25: 3.

31 Churchill R, Allen J, Denman S, et al. Do the attitudes and beliefs of young teenagers towards general practice influence actual consultation behaviour? Br J Gen Pract 2000; 50: 953-957.

32 Wight $\mathrm{D}$, Raab G, Henderson M, et al. The limits of teacher-delivered sex education: interim behavioural outcomes from a randomised trial. BMJ 2002; 324: 1430-1433.

33 http://www.msoc-mrc.gla.ac.uk/share/SHARE.html.

34 Henderson M, Butcher I, Wight D, et al. What explains betweenschool differences in rates of sexual experience? Soc Sci Med 2004 (in press).

35 Henderson M, Wight D, Raab G. Methodology for Balanced Randomisation: The SHARE Trial. Working Paper No. 11. Glasgow, UK: MRC Social and Public Health Sciences Unit, 2003

36 Henderson M, Wight D, Raab G, et al. Heterosexual risk behaviour among young teenagers in Scotland. J Adolesc 2002; 25: 483-494.

37 Wight $\mathrm{D}$, Henderson M. The diversity of young people's heterosexual behaviour. In: Burtney E, Duffy M (eds), Young People and Sexual Health: Individual, Social and Policy Contexts. London, UK: Palgrave Macmillan, 2004.

38 Rasbash J, Brown W, Healy M, et al. MLwiN - Development Test Version 1.2: Multilevel Models Project. London, UK: Institute of Education, 2002. http://multilevel.ioe.ac.uk/features/index.html.

39 Wight D, Williamson L, Henderson M. Parental influences on young people's sexual behaviour: a longitudinal study. J Adolesc 2004; (in press).

40 Snijders TAB, Bosker RJ. Multilevel Analysis: An Introduction to Basic and Advanced Multilevel Modeling. London, UK: Sage Publications, 1999.

41 Sustainable Development Directorate. Annex: Rural and Urban Areas: classifications used in this report. In: Indicators of Sustainable Development for Scotland: Progress Report 2004. Edinburgh, UK: Sustainable Development Directorate, Scottish Executive, 2004. http://www.scotland.gov.uk/publications/recent.aspx

42 Allen I. Family Planning and Pregnancy Counselling Projects for Young People. London, UK: Policy Studies Institute, 1991.

43 Jackson P, Plant Z. Mock sexual health clinics for school pupils. Health Educ 1997; 1: 16-18.

44 Meyrick J, Swann C. Reducing the Rate of Teenage Conceptions. An Overview of the Effectiveness of Interventions and Programmes Aimed at Reducing Unintended Conceptions in Young People. Aimed at Reducing Unintended Conceptions

45 Cromer BA, McCarthy M. Family planning services in adolescent pregnancy prevention: the views of key informants in four countries. Fam Plann Perspect 1999; 31: 287-293.

46 Thistle S. Secondary Schools and Sexual Health Services: Forging the Links. London, UK: National Children's Bureau for the Sex Education Forum, 2003.

47 Scottish Executive. Enhancing Sexual Wellbeing in Scotland: A Sexual Health and Relationships Strategy. Edinburgh, UK: Scottish Executive, 2003

48 Mellanby AR, Rees JB, Tripp JH. Peer-led and adult-led school health education: a critical review of available comparative research. Health Educ Res 2000; 15: 533-545.

49 Forrest S, Strange V, Oakley A. A comparison of students' evaluations of a peer-delivered sex education programme and teacher-led provision. Sex Educ 2002; 2: 195-214. 